\title{
Preparation, optimization, and in vitro simulated inhalation delivery of carvedilol nanoparticles loaded on a coarse carrier intended for pulmonary administration
}

\author{
This article was published in the following Dove Press journal: \\ International Journal of Nanomedicine \\ 12 October 2015 \\ Number of times this article has been viewed
}

\author{
Aly A Abdelbary' \\ Abdulaziz M Al-mahallawi' \\ Mohamed E Abdelrahim² \\ Ahmed MA Ali, \\ 'Department of Pharmaceutics \\ and Industrial Pharmacy, Faculty \\ of Pharmacy, Cairo University, Cairo, \\ ${ }^{2}$ Department of Clinical Pharmacy, \\ ${ }^{3}$ Department of Pharmaceutics, \\ Faculty of Pharmacy, Beni Suef \\ University, Beni Suef, Egypt; \\ ${ }^{4}$ Department of Pharmaceutics, \\ Faculty of Pharmacy, Taif University, \\ Taif, Saudi Arabia
}

\begin{abstract}
Carvedilol (CAR) is a potent antihypertensive drug but has poor oral bioavailability (24\%). A nanosuspension suitable for pulmonary delivery to enhance bioavailability and bypass first-pass metabolism of CAR could be advantageous. Accordingly, the aim of this work was to prepare CAR nanosuspensions and to use artificial neural networks associated with genetic algorithm to model and optimize the formulations. The optimized nanosuspension was lyophilized to obtain dry powder suitable for inhalation. However, respirable particles must have a diameter of $1-5 \mu \mathrm{m}$ in order to deposit in the lungs. Hence, mannitol was used during lyophilization for cryoprotection and to act as a coarse carrier for nanoparticles in order to deliver them into their desired destination. The bottom-up technique was adopted for nanosuspension formulation using Pluronic stabilizers (F127, F68, and P123) combined with sodium deoxycholate at 1:1 weight ratio, at three levels with two drug loads and two aqueous to organic phase volume ratios. The drug crystallinity was studied using differential scanning calorimetry and powder X-ray diffractometry. The in vitro emitted doses of CAR were evaluated using a dry powder inhaler sampling apparatus and the aerodynamic characteristics were evaluated using an Andersen MKII cascade impactor. The artificial neural networks results showed that Pluronic F127 was the optimum stabilizer based on the desired particle size, polydispersity index, and zeta potential. Results of differential scanning calorimetry combined with powder X-ray diffractometry showed that CAR crystallinity was observed in the lyophilized nanosuspension. The aerodynamic characteristics of the optimized lyophilized nanosuspension demonstrated significantly higher percentage of total emitted dose $(89.70 \%)$ and smaller mass median aerodynamic diameter $(2.80 \mu \mathrm{m})$ compared with coarse drug powder ( $73.60 \%$ and $4.20 \mu \mathrm{m}$, respectively). In summary, the above strategy confirmed the applicability of formulating CAR in the form of nanoparticles loaded on a coarse carrier suitable for inhalation delivery.
\end{abstract}

Keywords: aerodynamic diameter, freeze-drying, artificial neural networks, Pluronics, nanosizing, cascade impactor

\section{Introduction}

Pulmonary delivery is a needle-free route for systemic delivery of small molecule drugs. Because of the large surface area of the alveoli immediately accessible to drug, the lungs are suitable sites for absorption. The relatively low metabolic activity locally, lack of first-pass hepatic metabolism, and the elevated blood flow that rapidly distributes molecules throughout the body also confirm the suitability of lungs as an absorption site for drugs. ${ }^{1,2}$ Dry powder inhalers (DPIs) are among the most popular aerosol devices that are used for inhalation of drug formulations. DPIs are
Correspondence: Aly A Abdelbary Department of Pharmaceutics and Industrial Pharmacy, Faculty of Pharmacy, Cairo University, Kasr El Aini Street, Cairo II562, Egypt

Tel +20 II49005526

Email aly.abdelbary@pharma.cu.edu.eg 
advantageous compared to metered dose inhalers in that they do not require synchronization of inhalation and actuation. Moreover, they can deliver large drug doses, suitable for drugs that are unstable in solution form, easy to use, and safe to the environment. ${ }^{3}$ Nanoparticles in pulmonary drug delivery are considered a promising approach because of the uniform distribution of drug dose among the alveoli and enhanced solubility and dissolution rate of the drug. ${ }^{4}$ In addition, inhaled nanoparticles delivery systems are beneficial in reducing extrathoracic depositional drug losses by achieving almost zero deposition in the mouth-throat region. ${ }^{5}$ However, respirable particles must have a diameter of $1-5 \mu \mathrm{m}$ in order to deposit in the lungs. ${ }^{6}$ Nanosuspensions have been employed to enhance the dissolution rate of poor water-soluble drugs. ${ }^{7}$ Techniques used to produce drug nanosuspensions can be divided into two major classes: top-down and bottom-up technologies. ${ }^{8}$ Media milling and high-pressure homogenization are the most common methods of top-down approach. ${ }^{9}$ Despite being widely used, these techniques have limitations including long preparation time, difficulty in achieving a narrow size distribution, high energy input, and contamination. ${ }^{8,10}$ Bottom-up approaches are advantageous with respect to improving bioavailability by obtaining smaller particle sizes (PSs). ${ }^{10}$ The antisolvent precipitation process is a promising technique to prepare nanosuspensions, which is rapid to perform, cost-effective, and suitable for scaling up. ${ }^{11,12}$

Recently, artificial neural networks (ANNs) have been widely used to evaluate nonlinear processes in which classical statistical techniques often fail to produce a reliable model. ${ }^{13}$ ANNs take input and generate an output by computing the associated weights of input. Usually, a three-layer structure is formed in which an input layer takes independent (input) parameters, a hidden layer makes all the computations, and an output layer shows the dependent (output) parameters. ${ }^{14}$ Furthermore, they are considered powerful tools used for modeling and optimization of multivariable and complex domain data. ${ }^{15}$ ANNs do not need prior establishment of equations to describe relationship between input and output variables. ${ }^{16,17}$ In addition, ANNs can be used for historical data, and models generated can be updated with new experiments. ${ }^{18}$ ANNs combined with genetic algorithm also enable special operations such as "what-if" predictions and optimizations. ${ }^{19}$

Carvedilol (CAR) is a nonselective $\beta$-blocking agent that displays $\alpha_{1}$-adrenergic antagonism, resulting in a blood pressure reducing action through vasodilatation. CAR is classified as biopharmaceutics classification system class II drug with low solubility and high permeability. ${ }^{20}$ It is also subjected to a significant degree of first-pass metabolism. Hence, the absolute bioavailability of CAR has been estimated to be $24 \% .^{21,22}$ The enhancement of the aqueous solubility and the dissolution rate of CAR have been extensively studied. For example, the use of lipophilic solutions, ${ }^{23}$ formation of inclusion complex with cyclodextrin, ${ }^{24}$ the use of a self-emulsifying system, ${ }^{25}$ and preparation of solid dispersions ${ }^{26}$ have all been used to improve the solubility and dissolution rate of CAR. However, studies addressing its delivery through the pulmonary route using nanoparticles are rare, and none of these has linked the optimization of formulation to the in vitro simulated pulmonary delivery of the drug.

Therefore, this study aims to design and optimize stable CAR nanosuspensions adopting the antisolvent precipitationultrasonication method in order to improve drug dissolution rate, which will consequently enhance bioavailability. Pluronic stabilizers (F127, F68, and P123) combined with sodium deoxycholate (SDC) at 1:1 weight ratio were used at three levels with two drug loads and two aqueous to organic phase volume ratios. The modeling and optimization of nanosuspensions were performed using ANNs combined with genetic algorithm. Moreover, the optimized CAR nanosuspension formula was lyophilized in the presence of mannitol, which was intended to perform a dual action: cryoprotectant and coarse carrier for nanoparticles. ${ }^{27,28}$ The lyophilized powder was then incorporated in DPI intended for pulmonary administration. The in vitro emitted doses of CAR were evaluated using a DPI sampling apparatus with a critical flow controller and the aerodynamic characteristics were evaluated using an Andersen MKII cascade impactor (ACI).

\section{Materials}

CAR was obtained as a gift from Global Napi Pharmaceuticals (Giza, Egypt). Pluronics F127, F68, P123, and SDC were purchased from Sigma Chemical Co. (St Louis, MO, USA). Size 3 capsules suitable for the Aerolizer ${ }^{\circledR}$ DPI were obtained as free samples from Novartis Pharma (Cairo, Egypt). High-performance liquid chromatography (HPLC) grade acetonitrile, methanol, and $o$-phosphoric acid were purchased from Porlabo (Paris, France). The silicone fluid was obtained from Dow Corning Limited (Barry, Glamorgan, UK). Mannitol was purchased from Al-Nasr Chemical Co. (Cairo, Egypt). All other chemicals were of analytical grade.

\section{Methods}

\section{Preparation of CAR nanosuspensions}

CAR nanosuspensions were prepared by modifying the antisolvent precipitation-ultrasonication method mentioned 
in the literature. ${ }^{20}$ Briefly, CAR was dissolved completely in methanol to prepare the organic phase. The antisolvent phase was prepared by dispersing the stabilizers (Pluronic F127, Pluronic F68, and Pluronic P123) each combined with SDC (costabilizer) at 1:1 weight ratio in distilled water. Three levels of drug to stabilizer weight ratios (1:2,1:3, and 1:4), two drug loads (12.5 and $25 \mathrm{mg}$ ), and two aqueous to organic phase volume ratios (4:1 and 10:1) were used (Table 1). At a fixed temperature $\left(25^{\circ} \mathrm{C}\right)$, the organic solution was quickly injected by a syringe into the specified volume of antisolvent and the dispersion was simultaneously sonicated using an Elmasonic S40 water bath sonicator (ultrasonic frequency: $37 \mathrm{kHz}, 40 \mathrm{~W}$; Elma, Singen, Germany) for 15 minutes. Immediately, drug particles precipitated from the antisolvent. Then, the nanosuspensions were kept under reduced pressure in a rotary evaporator (Rotavapor, Type $\mathrm{R}$ 110, Büchi Labortechnik AG, Flawil, Switzerland) at $40^{\circ} \mathrm{C}$ for 15 minutes to remove methanol.

\section{Particle size, polydispersity index, and zeta potential of prepared nanosuspensions}

PS, polydispersity index (PDI), and zeta potential (ZP) of the prepared CAR nanosuspensions were measured by photon correlation spectroscopy using a Zetasizer Nano ZS-90 instrument (Malvern Instruments, Malvern, UK). An aliquot of the nanosuspension was diluted before the measurement. Measurements were performed in triplicate using $90^{\circ}$ scattering angle at $25^{\circ} \mathrm{C}$. The displayed results are the average value \pm standard deviation (SD).

\section{Modeling of nanosuspension formulae}

Nanosuspension formulae data set was composed of 36 records based on different input variables. These included drug load, stabilizer type, stabilizer hydrophilic-lipophilic balance (HLB), molecular weight of stabilizer, numbers of polyethylene oxide and polypropylene oxide units (PEO and PPO), ratio of hydrophilic to hydrophobic units, weight of stabilizer, weight ratio of drug to stabilizer, phase volume ratio (aqueous to organic), and the volume of aqueous phase (Table 1). The measured dependent variables included average PS, average PDI, and average ZP. Modeling and optimization of the data were carried out using ANNs-genetic algorithm software package (INForm V3.6; Intelligensys Ltd., UK) ${ }^{29}$ This modeling tool depends on a multilayer perceptron network embedded into the program that is responsible for model training, ${ }^{30}$ using the entered data to build up the cause-effect relationships between input variables (eleven formulation variables) and the output parameters

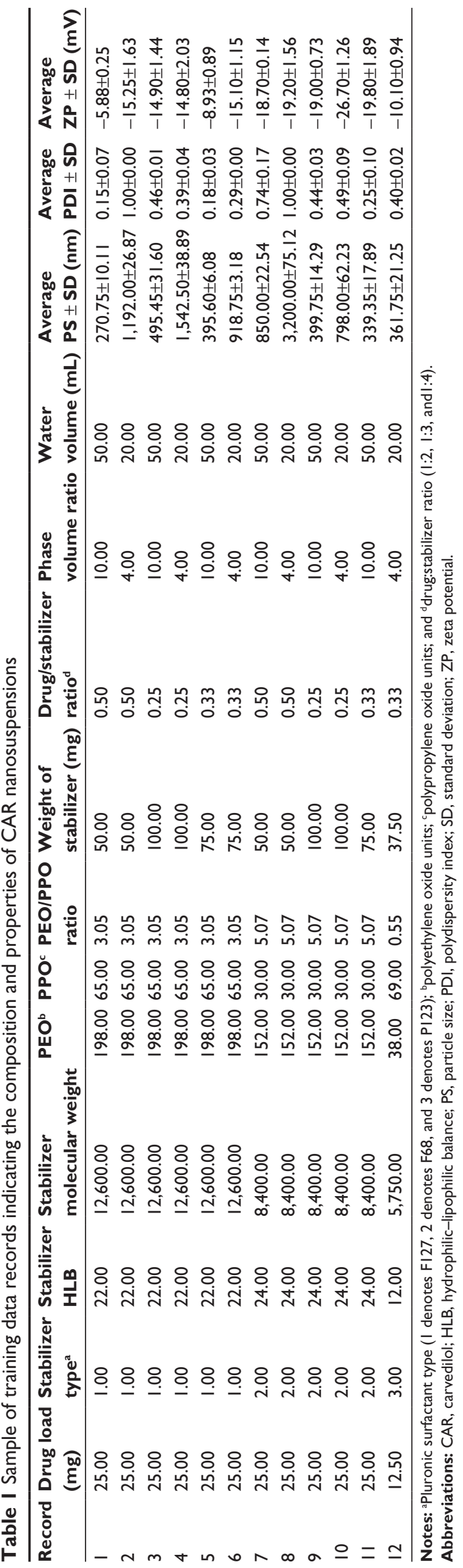


(PS, PDI, and ZP). The data set was divided into training records ( $80 \%)$, testing records $(10 \%)$, and validation records $(10 \%)$. Predictability of trained models was evaluated by the correlation coefficient $\left(R^{2}\right)$ values computed automatically during training, testing, and validation steps. ${ }^{30,31}$ High $R^{2}$ values $(>75 \%)$ indicated appropriate predictability of the trained model. ${ }^{14}$ The formula was derived from analysis of variance statistics generated by the modeling software:

$$
R^{2}=1-\frac{\sum_{i-1}^{n}\left(y_{i}-y_{i}^{*}\right)^{2}}{\sum_{i-1}^{n}\left(y_{i}-y_{i}^{-}\right)^{2}} \times 100
$$

where $y_{i}$ is the individual value of the dependent variable, $y_{i}^{*}$ the predicted value from the model, and $y_{i}^{-}$the mean of the dependent variable. In this formula, the numerator represents the sum of squares for the error term and the denominator represents the total sum of squares. ${ }^{3}$ The values of $R^{2}$ describe how much of the variance of the dependent variable is accounted for in the model. The ANN structure $\mathrm{I}(11)-\mathrm{H}(2)-\mathrm{O}(1)$ was used for model training (linking input and the output parameters). The default model training rapid back-propagation algorithm was composed of eleven nodes representing the input layer, two nodes in the hidden layer, and one node in the output layer.

The model transfer function (model activation function) was selected for input and output parameters as asymmetric sigmoid/linear. Further evaluation of the models was carried out by calculating the root mean-squared error (RMSE) values after training and testing for the measured parameters (PS, PDI, and ZP).

\section{Optimization of the prepared nanosuspensions}

After developing the predictive models for each parameter, optimization was carried out by setting the desired constraints on some process variables: setting the numbers of PEO and PPO units, molecular weight of stabilizer and its HLB as fixed values and the surfactant type, phase volume ratio, and the ratio of hydrophilic to hydrophobic units as integer values. The desired range for each of the output parameters was entered into the optimization screen. The desirability function was selected as tent in the model optimization window. The desired minimum and maximum values for the output parameters (PS, PDI, and ZP) were assigned as PS (250-300 $\mathrm{nm})$, PDI (0.1-0.3), and $\mathrm{ZP}(-2$ to $-10 \mathrm{mV})$ ranges in the optimization step. The model optimized solution (optimized formula) was generated based on previous knowledge gathered from training and testing and saved inside the model.

\section{Lyophilization of the optimized nanosuspension}

In order to obtain a dry powder suitable for inhalation, the model-optimized nanosuspension formula was lyophilized after preparation. First, the nanosuspension was poured into glass flasks and prefrozen using an ultra cold freezer (Revco $^{\mathrm{TM}}$, Thermo Scientific, Waltham, MA, USA) at $-80^{\circ} \mathrm{C}$ for 12 hours, then the samples were freeze-dried using a Flexi-Dry'M MP Freeze Dryer (SP Scientific, Stone Ridge, $\mathrm{NY}$, USA) at $-90^{\circ} \mathrm{C}$ and $380 \mathrm{mT}$ of pressure for 48 hours to yield dry nanoparticles in a powder form. Mannitol $(1 \% \mathrm{w} / \mathrm{v})$ was added into the dispersions prior to freezing as a cryoprotectant. In addition to cryoprotection, mannitol was intended to serve as a coarse carrier for the lyophilized nanoparticles during inhalation. The lyophilized powder was then sieved through a 100-mesh sieve and was left in a desiccator for characterization. PS, PDI, and ZP were reanalyzed for the lyophilized formula after reconstitution and the results were compared with those before lyophilization. Statistical analysis of data was performed using the software SPSS 19.0 (SPSS Inc., Chicago, IL, USA) applying one-way analysis of variance test, and the results were considered significantly different when $P$-values were $<0.05$.

\section{Morphology and surface characteristics of optimized nanosuspension}

The particle morphology of the reconstituted optimized nanosuspension formula was examined using transmission electron microscopy (TEM, H-600, Hitachi Ltd., Tokyo, Japan). The sample was dropped on copper-gold carbon grid and allowed to dry. The grid was then mounted in the instrument and photographs were taken at different magnifications..$^{32}$ In addition, the surface characteristics of the optimized lyophilized nanosuspension were observed using a scanning electron microscope (JXA-840; JEOL, Tokyo, Japan). The samples were gold-coated under vacuum and then examined. ${ }^{27}$

\section{Assessment of crystallinity of the freeze- dried nanosuspension}

Differential scanning calorimetry (DSC) samples of $\sim 5 \mathrm{mg}$ were weighed and analyzed in hermetically sealed aluminum pans. DSC was performed using a DSC822e Mettler Toledo differential scanning calorimeter (Mettler Toledo Inc., Columbus, $\mathrm{OH}, \mathrm{USA}$ ). Samples were heated at a scanning rate of $10^{\circ} \mathrm{C} \mathrm{min}^{-1}$ between $25^{\circ} \mathrm{C}$ and $300^{\circ} \mathrm{C}$ using nitrogen as blanket gas. ${ }^{33,34}$ Empty aluminum pan was used as a standard reference. Powder X-ray diffraction (PXRD) studies 
were conducted using an X-ray diffractometer (MD-10 mini diffractometer; MTI Corporation, Richmond, CA, USA) using $\mathrm{Cu} \mathrm{K} 2 \alpha$ rays $(\lambda=1.54056 \AA$ ) with a voltage of $25 \mathrm{kV}$ and a current of $30 \mathrm{~mA}$, in a flat plate $\theta / 2 \theta$ geometry, over the $2 \theta$ range of $5^{\circ}-75^{\circ}$. A sample of $60 \mathrm{mg}$ was placed in the sample holder groove and packed tightly. ${ }^{35}$

\section{Determination of saturated solubility}

The saturation solubilities of the previously sieved coarse CAR powder and the lyophilized nanosuspension formula were determined by adding an excess amount of the powder in a vial containing $5 \mathrm{~mL}$ Sorenson's phosphate buffer $\mathrm{pH} 6.8$. Shaking was performed using thermostatically controlled shaking water bath (Precision Scientific, Chicago, IL, USA) at $37^{\circ} \mathrm{C}$ till equilibrium (48 hours). Samples were taken and filtered using a $0.1 \mu \mathrm{m}$ membrane filter (Whatman Inc., Clifton, NJ, USA) and the filtrate was analyzed spectrophotometrically at a $\lambda_{\max }$ of $284 \mathrm{~nm} .{ }^{36}$ The experiment was conducted in triplicate for each formula and the mean values \pm standard deviations were calculated. Statistical analysis of data was performed using the software SPSS 19.0 (SPSS Inc.).

\section{In vitro dissolution study}

In this test, a comparative dissolution study was performed between an amount of lyophilized nanosuspension equivalent to $12.5 \mathrm{mg}$ CAR and the previously sieved coarse drug powder. This was carried out using USP II dissolution apparatus (Hanson Research Corp., Chatsworth, CA, USA) containing $900 \mathrm{~mL}$ Sorenson's phosphate buffer pH 6.8 as dissolution medium at $37^{\circ} \mathrm{C}$ and stirred at $50 \mathrm{rpm} .{ }^{37}$ Dissolution samples were withdrawn at $5,10,15,20,30,45,60$, and 90 minutes, with replacement of an equal volume of dissolution medium. The samples were filtered and analyzed using an UV spectrophotometer as mentioned earlier. All samples were analyzed in triplicate. Release rate was expressed by the mean dissolution time (MDT), which was calculated from the equation:

$$
M D T=\frac{\sum_{j=1}^{n} t_{j}^{*} \Delta M_{j}}{\sum_{j=1}^{n} \Delta M_{j}}
$$

where $j$ is the sample number, $n$ the number of dissolution samples, $t_{j}^{*}$ the time at midpoint between $t$ and $t_{j-1}$, and $\Delta M_{j}$ the additional amount of drug dissolved between $t$ and $t_{j-1}{ }^{38}$ Also, similarity factor $\left(f_{2}\right)$ was calculated for multiple comparisons between the obtained release profiles utilizing the following equation:

$$
f_{2}=50 \times \log \left\{\left[1+\left(\frac{1}{n}\right) \sum_{j=1}^{n}\left|R_{j}-T_{j}\right|^{2}\right]^{-0.5} \times 100\right\}
$$

where $n$ is the sampling number, and $R$ and $T$ are the percent dissolved of the reference and test products, respectively, at each time point $j .^{38}$

\section{Simulated in vitro inhalation}

An amount of the optimized lyophilized nanosuspension equivalent to $2,000 \mu \mathrm{g}$ CAR was placed in capsules (size 3) suitable for the Aerolizer ${ }^{\circledR}$ DPI (Novartis Pharma) to be tested for the aerodynamic characteristics. A physical mixture of coarse CAR powder $(2,000 \mu \mathrm{g})$ combined with Pluronic F127, SDC, and mannitol was also sieved through a 100-mesh sieve and used for comparison. The emitted dose from the Aerolizer ${ }^{\circledR}$ was measured using a DPI sampling apparatus with a critical flow controller model TPK (Copley Scientific Ltd., Nottingham, UK). The final filter was a $47 \mathrm{~mm} \mathrm{~A} / \mathrm{E}$ fiber glass filter disks (Pall Corporation, USA). The inhalation flow through the mouthpiece of the Aerolizer ${ }^{\circledR}$ was set at $60 \mathrm{~L} \mathrm{~min}^{-1}$ with a flow duration of 4 seconds to allow an inhaled volume of $4 \mathrm{~L}$ of air to be withdrawn through the inhaler. The basic methodology is described in Appendix XIIF of the BP, ${ }^{39} 2.9 .18$ of the EP, ${ }^{40}$ and 601 of the USP. ${ }^{41}$ The emitted dose from the Aerolizer ${ }^{\circledR}$ was measured $(n=5)$ by collecting one individual dose each time the Aerolizer ${ }^{\circledR}$ was loaded and operated.

Following dose emission into the apparatus, the sampling unit, including filter, was washed with a mixture of methanol and acetonitrile (80:20 ratio) and sonicated for 3 minutes. The solutions were then collected and made up to a volume of $100 \mathrm{~mL}$. The amount of drug was determined using a previously validated HPLC method. ${ }^{42}$ The method included a mobile phase composed of methanol:acetonitrile: $1 \%$ o-phosphoric acid 80:18:2 v/v/v with a pH of 6.2 using Chromosil $\mathrm{C}_{18}$ analytical column $(250 \mathrm{~mm} \times 4.6 \mathrm{~mm}, 5 \mu \mathrm{m})$. A sample of $20 \mu \mathrm{L}$ was injected at ambient temperature for 6 minutes run time with a flow rate of $1 \mathrm{~mL} \mathrm{~min}^{-1}$ and effluents were identified at $240 \mathrm{~nm}$ with a UV detector (RF-551; Shimadzu, Kyoto, Japan).

The aerodynamic PS characterization was evaluated using the ACI. The ACI was assembled with modification plates for a flow rate of $60 \mathrm{~L} \mathrm{~min}^{-1}$ with a flow duration of 4 seconds, hence stages 0 and 7 were replaced by -0 and -1 on the top of the impactor. The collection plates were then sprayed with silicone fluid (Releasil B silicone spray; Dow Corning Limited) and then allowed to dry for at least 1 hour prior to the analysis. The ACI was assembled with $10 \mathrm{~mL}$ of the 
previously mentioned methanol/acetonitrile mixture placed in the preseparator and the final filter was of type GF 50 (Copley Scientific Ltd.). The flow was measured using an electronic digital flow meter (MKS Instruments, USA) and a critical flow controller model TPK (Copley Scientific Ltd.). Parafilm M laboratory film (Pechiney Plastic Packaging Company, Chicago, IL, USA) was used to seal the apparatus.

For each determination, only one dose was discharged into the ACI. Three determinations from each formula were made, according to a randomization procedure. Each stage of the ACI was rinsed with a specified volume of the previously mentioned methanol/acetonitrile mixture. The washing procedure was the same as that described for the total dose emission. The amounts deposited on each stage were determined by the previously mentioned HPLC method.

\section{Aerodynamic data analysis}

The total dose emission was determined as the total amount of drug ex-mouthpiece. This was reported with respect to the nominal emitted dose. Using the ACI with a flow of $60 \mathrm{~L} \mathrm{~min}^{-1}$, the effective cutoff diameter of each stage was fixed at $60 \mathrm{~L} \mathrm{~min}^{-1}$ flow. ${ }^{43,44}$ The fine particle dose (FPD) was the amount of particles corresponding to a size less than $5 \mu \mathrm{m}$. The fine particle fraction \% was the FPD expressed as a percentage of the total amount deposited into the throat and stages of the ACI (the dose exited the mouthpiece). The mass median aerodynamic diameter (MMAD) was obtained from a plot of the logarithm of the percentage less than a stated size on a probability scale against the logarithm of the effective cutoff diameter of the stage, and this was carried out using the Copley Inhaler Testing Data Analysis Software. The MMAD was the diameter separating the mass of the particles equally by $50 \%$. The geometric standard deviation was a measure of the polydispersity of the PS distribution. Geometric standard deviation was calculated as the square root for the size corresponding to $84.13 \%$ less than the stated size divided by the square root of the size corresponding to $15.87 \% .{ }^{41}$ The aerodynamic results were compared using a paired $t$-test with a significance level set at $P<0.05$.

\section{Results and discussion Modeling of nanosuspension formulae}

Data collected from the experimental design (36 records) were subjected to the modeling step and resulted in a good model with high predictability as indicated by its high model training and testing regression $R^{2}$ values being $94.02 \%$ and 93.40\% for average PS, $92.77 \%$ and $90.44 \%$ for average PDI, and $98.49 \%$ and $82.28 \%$ for average ZP, respectively (Table 2). From model statistics shown in Table 2, model trustability can be emphasized by calculating the RMSE and comparing these values between model training and testing. The RMSE values obtained from model training and testing

Table 2 ANOVA statistics obtained from model training and testing data sets

\begin{tabular}{|c|c|c|c|c|c|c|}
\hline Property & Source of variation & Sum of squares & DF $^{\mathrm{a}}$ & MSE $^{\mathrm{b}}$ & RMSEc $^{\mathrm{c}}$ & CF ratiod \\
\hline \multirow[t]{7}{*}{ Average PS (nm) } & Model & $41,682,100$ & 25 & $\mathrm{I}, 667,284.00$ & $1,291.23$ & 3.28 \\
\hline & Error & $2,543,100$ & 5 & $508,620.00$ & 713.18 & \\
\hline & Total & $44,225,200$ & 30 & & & \\
\hline & Covariance term & Sum of errors & & & & \\
\hline & 526,462 & 13.7687 & & & & \\
\hline & Train set $R^{2}$ & $94.02 \%$ & & & & \\
\hline & Test set $R^{2}$ & $93.40 \%$ & & & & \\
\hline \multirow[t]{7}{*}{ Average PDI } & Model & 913.71 & 25 & 36.54 & 6.04 & 2.63 \\
\hline & Error & 69.5449 & 5 & $13.9 \mid$ & 3.73 & \\
\hline & Total & 962.321 & 30 & & & \\
\hline & Covariance term & Sum of errors & & & & \\
\hline & -20.9333 & -7.04133 & & & & \\
\hline & Train set $R^{2}$ & $92.77 \%$ & & & & \\
\hline & Test set $R^{2}$ & $90.44 \%$ & & & & \\
\hline \multirow[t]{7}{*}{ Average ZP (mV) } & Model & 968.07 & 25 & 38.72 & 6.22 & 12.89 \\
\hline & Error & 15.02 & 5 & 3.00 & 1.73 & \\
\hline & Total & 996.29 & 30 & & & \\
\hline & Covariance term & Sum of errors & & & & \\
\hline & 13.198 & -0.181534 & & & & \\
\hline & Train set $R^{2}$ & $98.49 \%$ & & & & \\
\hline & Test set $R^{2}$ & $82.28 \%$ & & & & \\
\hline
\end{tabular}

Notes: aDegrees of freedom; 'bean sum of squared errors; 'root mean-squared error; ${ }^{\mathrm{d}}$ computed $\mathrm{F}$ ratio.

Abbreviations: ANOVA, analysis of variance; PDI, polydispersity index; PS, particle size; ZP, zeta potential. 
for PS, PDI, and ZP were 1291.23 and 713.18, 6.04 and 3.73 , and 6.22 and 1.73 , respectively. The observed lower values of test data RMSE than those of training indicate high predictability and trustability of the obtained model..$^{45}$ In Table 3, the model-generated statistics for the validation data set indicated high $R^{2}$ values for unseen data being 85.18 , 88.03 , and $92.93 \%$ for PS, PDI, and ZP, respectively. Higher $F$ ratio values and lower RMSE also support the significance of the obtained models for the three parameters.

\section{Response surface plots}

The relationships between the independent and dependent variables were summarized by the model in $3 \mathrm{D}$ response surface plots demonstrating the effects of two independent variables at average levels of other variables on the output parameters (Figures 1-3). The results of response surface plots showed that the most effective formulation variables that directly affect the PS of the nanosuspensions included stabilizer type, number of PPO units, weight of stabilizer, and the ratio of hydrophilic to hydrophobic units (Figure 1). The effect of the type of stabilizer on PS can be explained by the response surface plot (Figure 1A) showing that the PS values were low at stabilizer type 1 (Pluronic F127), high at stabilizer type 2 (Pluronic F68), and low at stabilizer type 3 (Pluronic P123). The ratio of hydrophilic to hydrophobic units (PEO/PPO) demonstrated increasing effects on PS moving from 0.55 to 3.05 and finally to 5.07 corresponding to Pluronics P123, F127, and F68, respectively (Figure 1B).
However, the increase in PPO units from 30 (F68) through 65 (F127) and finally to 69 (P123) was found to have prominent decreasing effect on the PS, which is consistent with the effects of stabilizer type. Also, the increased weight of stabilizer led to a decrease in the size (Figure 1C). The drug to stabilizer ratio and phase volume ratio were found to have increasing and decreasing effects on PS, respectively (Figure 1D). The decrease in PS with increased levels of phase volume ratio might be due to the formation of more nucleation sites per unit volume of the antisolvent (aqueous phase). This caused the precipitation of less drug molecules per nucleation site and consequently decreased PS. ${ }^{46}$ Moreover, the decrease in PS with an increased weight of stabilizer could be attributed to the decrease in surface tension, which facilitated the size reduction and stabilized the formed nanoparticles with inhibition of aggregation. ${ }^{27}$ It is well known that the conformation of physically adsorbed triblock copolymers (Pluronics) depends on the hydrophobicity of the sorbent surface. ${ }^{47}$ The hydrophobic PPO block anchors to the hydrophobic surface, leaving the PEO chains extending in the aqueous phase, and when the number of the adsorbed polymeric chains is sufficiently high, a brush conformation is formed. ${ }^{47,48}$ The adsorption of a series of Pluronics on polystyrene colloids was studied to probe the effect of PEO chain length and the hydrophilic/hydrophobic block length ratio on the adsorption characteristics. ${ }^{49}$ It was found that the surface concentration of Pluronics on polystyrene colloids is determined by the size of the hydrophobic PPO block,

Table 3 ANOVA statistics obtained from model validation data sets

\begin{tabular}{|c|c|c|c|c|c|c|}
\hline Property & Source of variation & Sum of squares & $\mathbf{D F}^{\mathrm{a}}$ & MSE $^{b}$ & RMSEc $^{c}$ & CF ratio ${ }^{d}$ \\
\hline \multirow[t]{6}{*}{ Average PS (nm) } & Model & $10,736,667$ & 25 & 429,467 & 655.33 & 4.83 \\
\hline & Error & $\mathrm{I}, 867,767$ & -21 & 88,941 & 298.23 & \\
\hline & Total & $12,604,433$ & 4 & & & \\
\hline & Covariance term & Sum of errors & & & & \\
\hline & $-898,333.3$ & -370.45 & & & & \\
\hline & Validation set $R^{2}$ & $85.18 \%$ & & & & \\
\hline \multirow[t]{6}{*}{ Average PDI } & Model & 0.33797833 & 25 & 0.0135 & 0.12 & 6.18 \\
\hline & Error & 0.04594167 & -21 & 0.0022 & 0.05 & \\
\hline & Total & 0.38392 & 4 & & & \\
\hline & Covariance term & Sum of errors & & & & \\
\hline & -0.0495858 & -0.344973 & & & & \\
\hline & Validation set $R^{2}$ & $88.03 \%$ & & & & \\
\hline \multirow[t]{6}{*}{ Average ZP (mV) } & Model & 152.285 & 25 & 6.0914 & 2.47 & 11.04 \\
\hline & Error & | I.59| & -21 & 0.5519 & 0.74 & \\
\hline & Total & 163.876 & 4 & & & \\
\hline & Covariance term & Sum of errors & & & & \\
\hline & -3.49 & -42.25 & & & & \\
\hline & Validation set $R^{2}$ & $92.93 \%$ & & & & \\
\hline
\end{tabular}

Notes: a'Degrees of freedom; ${ }^{b}$ mean sum of squared errors; 'root mean-squared error; 'computed F ratio. Abbreviations: ANOVA, analysis of variance; PDI, polydispersity index; PS, particle size; ZP, zeta potential. 
A

Average PS (nm) (Z)

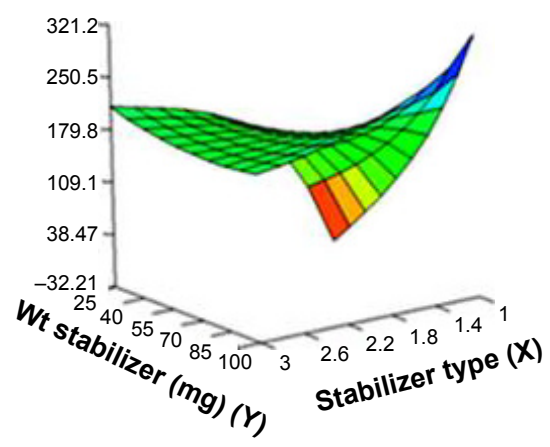

C

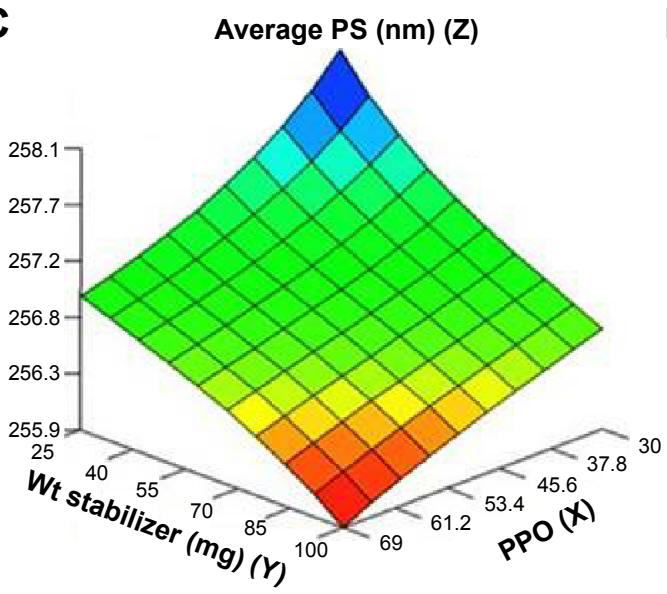

B

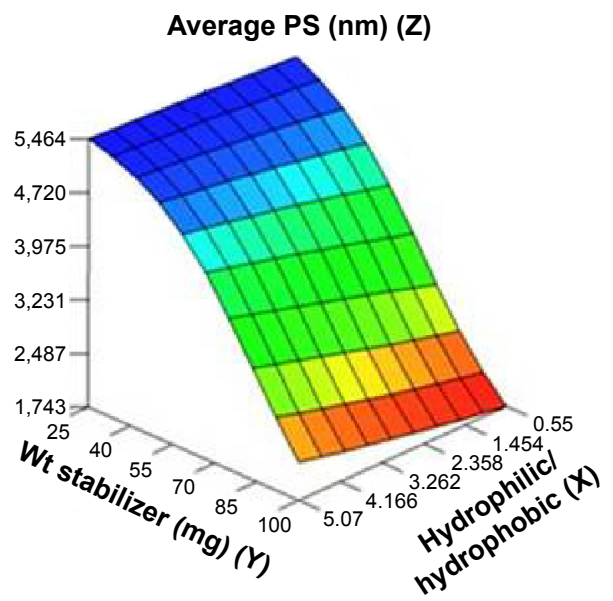

D

Average PS (nm) (Z)

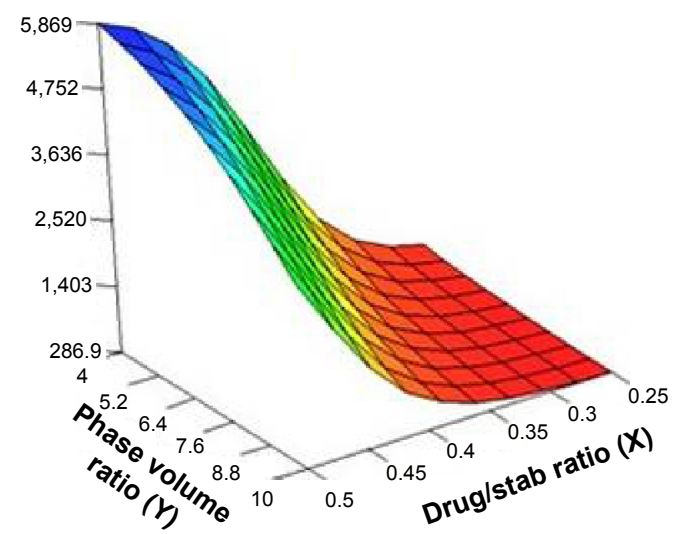

Figure I Response surface plots showing influence of some formulation variables on nanosuspension average particle size: (A) stabilizer type and wt of stabilizer; (B) ratio of hydrophilic to hydrophobic units and wt of stabilizer; (C) number of PPO units and wt of stabilizer; (D) drug/stab ratio and phase volume ratio.

Abbreviations: PS, particle size; PPO, polypropylene oxide; stab, stabilizer; Wt, weight.

independent of the size of hydrophilic PEO block. Hence, the hydrophobicity of the stabilizer is believed to have a major role in stable polymer adsorption onto the hydrophobic drug surfaces by forming high surface concentrations. This was confirmed by the above findings, which showed that the most hydrophobic stabilizer P123 (69 PPO units) demonstrated the smallest nanoparticle size, followed by the medium hydrophobic F127 (65 PPO units), and the largest size was demonstrated by the least hydrophobic F68 (30 PPO units). Hence, the important descriptive factors of stabilizers that enabled better understanding of the effects of the structure of each stabilizer on PS were the stabilizer type, the ratio of hydrophilic to hydrophobic units, and the number of PPO units.

For PDI, the important factors included phase volume ratio, water volume, molecular weight of stabilizer, and drug load (Figure 2A-D). The increased levels of phase volume ratio and water volume decreased the PDI. This might be explained by the formation of more nucleation sites per unit volume of the antisolvent as mentioned earlier. Hence, less drug molecules precipitated per nucleation site and a more uniform distribution for the PS was obtained resulting in lower PDI. ${ }^{46}$ On the other hand, the increase in molecular weight of stabilizer resulted in an increase in PDI. This could be attributed to the less kinetically restricted adsorption process of the stabilizers onto the drug surfaces that was enabled when the molecular weight of the stabilizer decreased. ${ }^{9}$ Hence, the opposite was expected to happen when the molecular weight increased resulting in less uniform distribution for the PS (higher PDI). Moreover, the unexpected limited decrease in average PDI with increased drug load from 12.5 to $25 \mathrm{mg}$ observed in Figure 2B could be ascribed to the limited scatter of drug load input variable between formulations. It may also be due to the sensitivity of PDI to other possible unmeasured variables such as lag time at the time of measurement. 
A

Average PDI (Z)

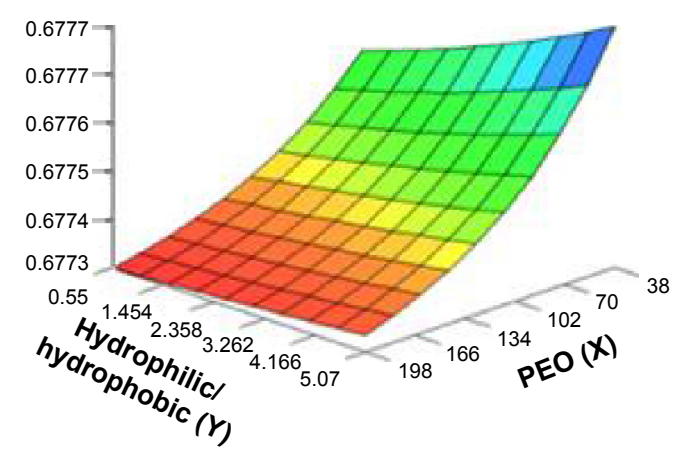

C

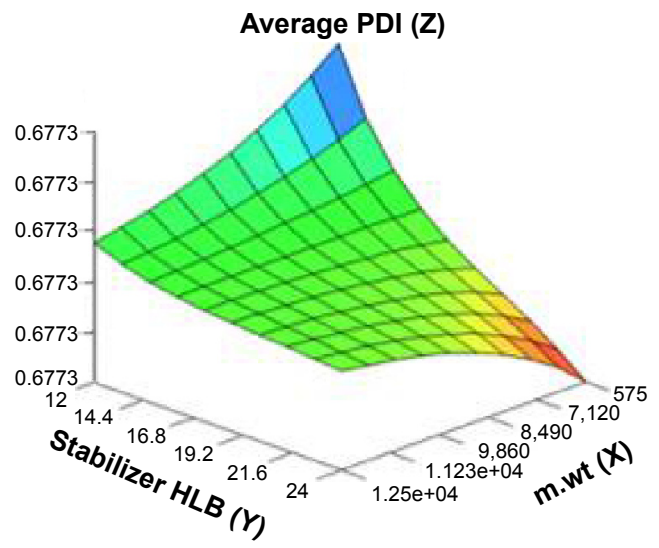

B

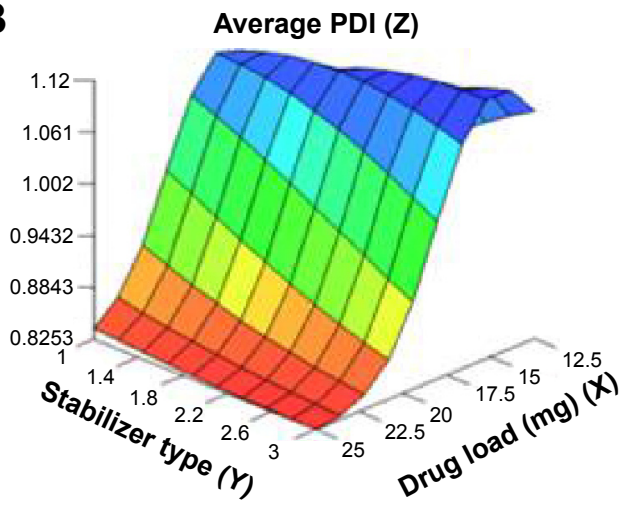

D

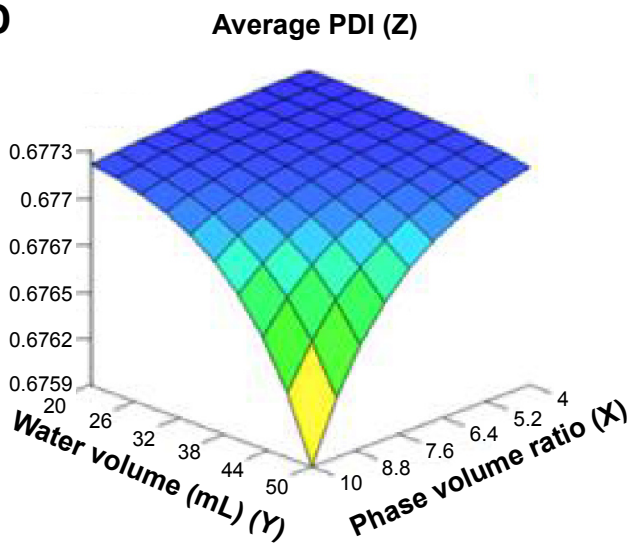

Figure 2 Response surface plots showing influence of some formulation variables on nanosuspension average polydispersity index: (A) number of PEO units and ratio of hydrophilic to hydrophobic units; (B) drug load and stabilizer type; (C) m.wt and stabilizer HLB; (D) phase volume ratio and water volume.

Abbreviations: PDI, polydispersity index; PEO, polyethylene oxide; m.wt, molecular weight; HLB, hydrophilic-lipophilic balance.

The ZP (Figure 3A-D) was found to increase by increased ratio of hydrophilic to hydrophobic units. It had an optimum value (as an absolute value) at a medium ratio of $\mathrm{PEO} / \mathrm{PPO}$ of 3.05 and drug/stabilizer ratio of 0.35 (Figure 3A). Stabilizer HLB and molecular weight appeared to be less effective variables on optimum ZP. The decrease in ZP with an increase in the thickness of the adsorbed polymer layer is due to the outward shift of the slipping plane, at which ZP is measured..$^{50}$ According to Gouy-Chapman theory, the slipping plane is moved to a point further out from the surface where the charge density is much smaller than on the surface resulting in lower ZP. This explains the lower ZP (as an absolute value) of Pluronic F127 nanosuspensions (having higher thickness of adsorbed polymer layer due to higher number of PEO units) compared with Pluronic F68 nanosuspensions (Table 1; Figure 3C).$^{51}$ In addition, the small values of $\mathrm{ZP}$ obtained in the case of Pluronic P123 might be due to the adsorption of high concentration of this highly hydrophobic stabilizer on the nanoparticles surface, which decreased the adsorption of ions and consequently ZP as shown by the effect of its PPO units (69) on ZP in Figure 3D. The weight of stabilizer in the range of 45-55 $\mathrm{mg}$ appeared to have an optimum level of ZP while, at lower and higher levels, the $\mathrm{ZP}$ increased (Figure 3D).

\section{Model optimization}

The obtained predictive models for nanosuspension formulae were tested for optimization of the three dependent variables (output parameters). The optimization stage resulted in an optimized solution (suggested nanosuspension formula) for the entered desired ranges of the variables. In this optimized solution, an average PS of $280.52 \mathrm{~nm}$, an average PDI of 0.25 , and an average $\mathrm{ZP}$ of $-9.50 \mathrm{mV}$ were obtained from a formula containing $23 \mathrm{mg}$ CAR, $48.80 \mathrm{mg}$ Pluronic F127, a phase volume ratio of 9, and a total aqueous phase volume of $45 \mathrm{~mL}$ (Table 4). When this theoretical suggestion was converted to the experimental analog, the resulting nanosuspension had similar attributes to those of the optimized solution. Insignificant differences for PS, PDI, and ZP were detected $(P>0.05)$ between model-suggested nanosuspension and the experimentally prepared counterpart. The prepared optimized nanosuspension was then 
A

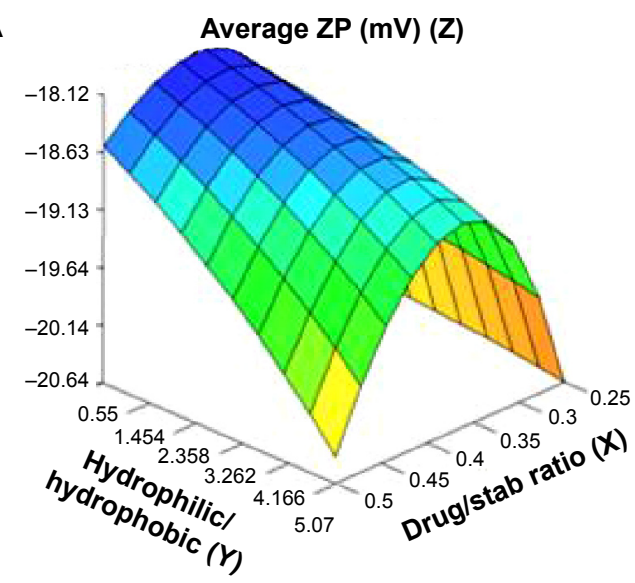

C

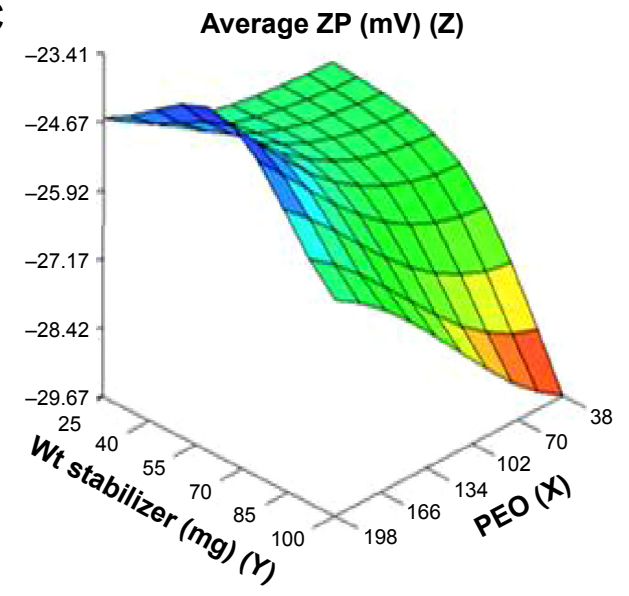

B

Average ZP (mV) (Z)

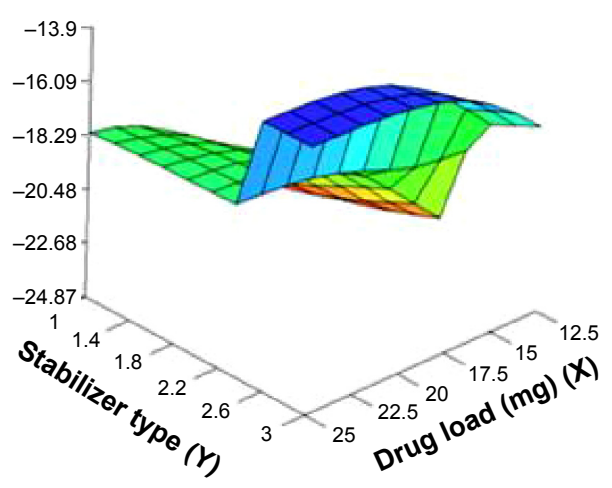

D

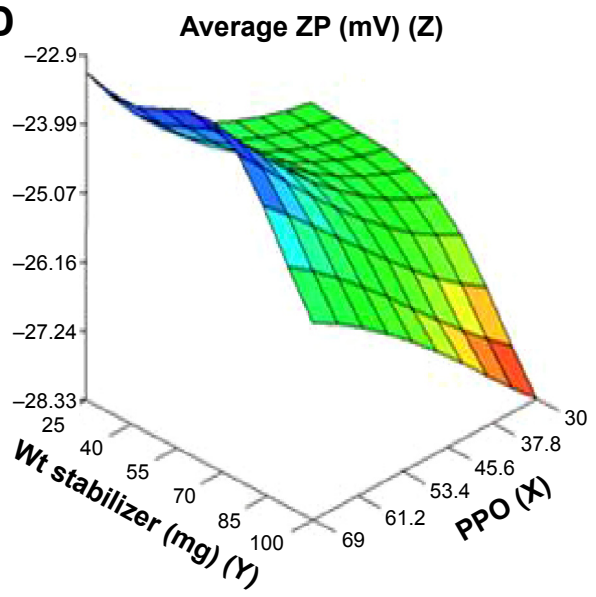

Figure 3 Response surface plots showing influence of some formulation variables on nanosuspension average zeta potential: (A) drug/stab ratio and ratio of hydrophilic to hydrophobic units; (B) drug load and stabilizer type; (C) number of PEO units and wt of stabilizer; (D) number of PPO units and wt of stabilizer.

Abbreviations: ZP, zeta potential; PPO, polypropylene oxide; PEO, polyethylene oxide; stab, stabilizer; Wt, weight.

subjected to freeze-drying followed by reconstitution. The properties of the reconstituted lyophilized nanosuspension were compared with those of the freshly prepared nanosuspension. The PS demonstrated an increase from 277.90 to $316.40 \mathrm{~nm}$. This difference was found to be statistically insignificant $(P>0.05)$. Also, insignificant differences were detected for the PDI and ZP before and after the lyophilization process $(P>0.05)$ as shown in Table 4 . The results confirmed the protective effect of mannitol on nanoparticles agglomeration. ${ }^{27}$
It is worth mentioning that although the modeling results demonstrated that Pluronic P123 showed minimum PS and ZP compared with the other two stabilizers (F127 and F68), yet the optimized solution generated by the model in optimization step suggested Pluronic F127 rather than P123. This selection made by the model may be attributed to two reasons: first, model consideration of interaction between variables at certain levels, and second, the model collectively calculates the input that would achieve the three parameters (PS, PDI, and ZP) all together as the desired output. Hence, the model

Table 4 Model-generated optimized solution and its analogous fresh and lyophilized experimental nanosuspensions

\begin{tabular}{|c|c|c|c|c|c|c|c|c|c|c|}
\hline \multirow[t]{3}{*}{ Solution } & \multirow[t]{3}{*}{ Desirability } & \multicolumn{6}{|c|}{ Nanosuspension independent variables (inputs) } & \multicolumn{3}{|c|}{$\begin{array}{l}\text { Dependent variables } \\
\text { (output properties) }\end{array}$} \\
\hline & & $\mathbf{X I}$ & $\mathbf{X} 2$ & $\mathbf{X 8}$ & $\mathbf{X 9}$ & $\mathbf{x} 10$ & $\mathbf{X I I}$ & Y I & Y2 & Y3 \\
\hline & & $\begin{array}{l}\text { Drug load } \\
(\mathrm{mg})\end{array}$ & $\begin{array}{l}\text { Stabilizer } \\
\text { type }\end{array}$ & $\begin{array}{l}\text { Weight of } \\
\text { stabilizer (mg) }\end{array}$ & $\begin{array}{l}\text { Drug/stabilizer } \\
\text { ratio }\end{array}$ & $\begin{array}{l}\text { Phase volume } \\
\text { ratio }\end{array}$ & $\begin{array}{l}\text { Water volume } \\
(\mathrm{mL})\end{array}$ & PS (nm) & PDI & $\mathbf{Z P}(\mathrm{mV})$ \\
\hline Population I & 1.00 & 23 & I & 48.80 & 0.47 & 9 & 45 & 280.52 & 0.25 & -9.50 \\
\hline Fresh NS & NA & 23 & I & 48.80 & 0.47 & 9 & 45 & 277.90 & 0.19 & -10.38 \\
\hline Lyophilized NS & NA & 23 & I & 48.80 & 0.47 & 9 & 45 & 316.40 & 0.20 & -8.94 \\
\hline
\end{tabular}

Abbreviations: NS, nanosuspension; PS, particle size; PDI, polydispersity index; ZP, zeta potential; NA, not applicable. 


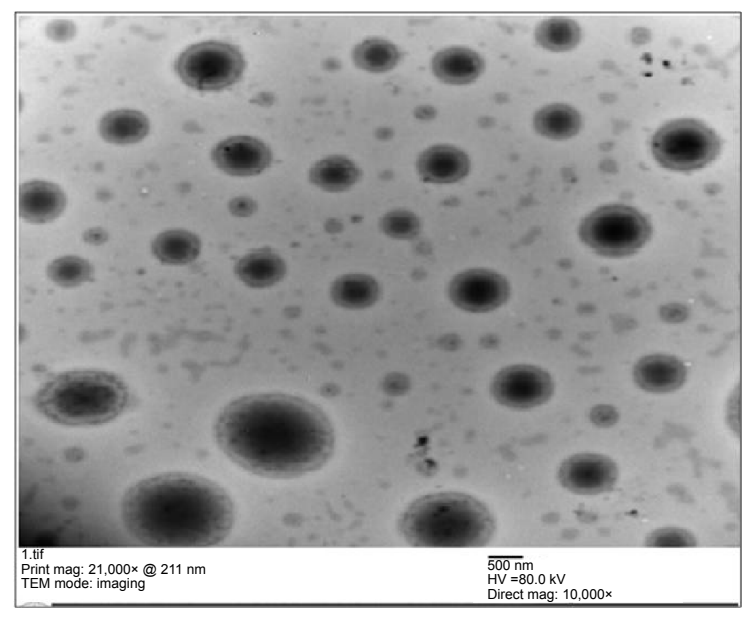

Figure 4 Transmission electron micrograph for the optimized carvedilol nanosuspension.

tries to fulfill the desired output parameters entered into the model optimization screen by testing all levels of the input variables with high desirability and minimum bias.

\section{Morphology and surface characteristics}

The optimized nanosuspension showed typical TEM images for nanoparticles at a direct magnification of $10,000 \times$. Spherical CAR nanoparticles with variable diameters are shown in Figure 4. The boundaries of the nanoparticles appeared less dark than the core, which suggests the adsorption of a thick layer of the surfactant stabilizer molecules at the surface of the particles. In addition, the PS shown by the TEM micrograph was in good agreement with that obtained by photon correlation spectroscopy. ${ }^{52}$ The scanning electron micrograph images of the freeze-dried nanosuspension showed smooth surface spherical nanoparticles embedded in a large hairy matrix of mannitol (Figure 5).

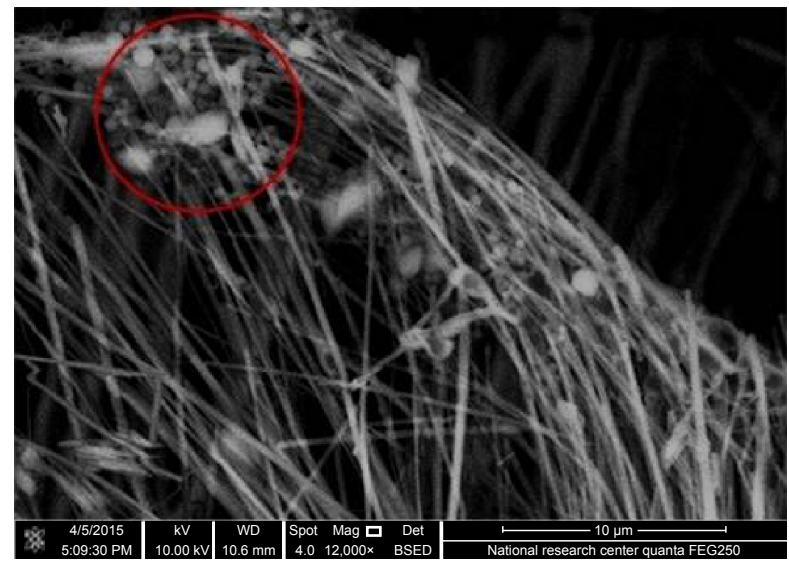

Figure 5 Scanning electron micrograph of freeze-dried nanosuspension showing dispersed spherical nanoparticles inside a crystalline matrix of cryoprotectant (red circle).

\section{Solid-state characterization (DSC and PXRD)}

Samples of individual ingredients, physical mixture, as well as the optimized freeze-dried nanosuspension formula were subjected to solid-state characterization using DSC and PXRD. The DSC curve for the coarse CAR (Figure 6) showed a sharp melting endotherm at $115.47^{\circ} \mathrm{C}$ indicating the crystalline nature of the drug in accordance with data in the literature. ${ }^{25}$ Pluronic F127 showed low melting endotherm at $54.86^{\circ} \mathrm{C}$. $^{7}$ SDC demonstrated a broad endotherm probably because of the loss of water molecules followed by an exothermic recrystallization peak at $214^{\circ} \mathrm{C} .{ }^{53}$ Mannitol demonstrated a sharp melting endotherm at $167^{\circ} \mathrm{C}$, which shows that it is a highly crystalline component. The drug characteristic peak disappeared in the thermogram of freeze-dried nanosuspension and was found with decreased intensity in the thermogram of physical mixture. This might be referred to the dilution effect of the drug with the excipients, especially in the presence of a wide shoulder for SDC that might cover the drug peak. In addition, there is a possibility for decrease or disappearance of drug crystallinity. However, these findings showed that DSC was not a discriminative tool for explaining the changes that occurred in drug crystals during preparation. ${ }^{27}$

The PXRD patterns shown in Figure 7 indicated that CAR exhibited three sharp characteristic diffraction lines at $2 \theta$ of $5.8^{\circ}, 14.7^{\circ}$, and $24.5^{\circ}$ and multiple short lines between $2 \theta$ of $10^{\circ}$ and $30^{\circ}$. Pluronic F127 demonstrated two reflections at $2 \theta$ of $19^{\circ}$ and $23^{\circ}$. SDC showed a characteristic halo for an amorphous structure, while mannitol showed a crystalline diffraction pattern with characteristic lines of increasing height at $2 \theta$ of $5^{\circ}, 10^{\circ}, 14^{\circ}, 18^{\circ}$, and $22^{\circ}$. The physical mixture showed a summation of diffraction lines mostly typical to parent crystalline components. The freezedried nanosuspension demonstrated two sharp diffraction lines between $2 \theta$ of $9.6^{\circ}$ and $20.2^{\circ}$ with two short lines in between $2 \theta$ of $14^{\circ}$ and $18^{\circ}$. This pattern is mostly similar to the characteristic diffraction lines observed for crystalline CAR. However, the diffraction lines were shifted to higher $2 \theta$ values possibly because of a new polymorphic form (IV), which was reported in the literature to be formed during antisolvent precipitation of crystalline CAR..$^{54}$ The new sharp diffraction line observed in the nanosuspension pattern at $72^{\circ}$ appeared to belong to SDC. This reflection line may confirm that SDC molecules had undergone crystallization during the freeze-drying process. In summary, the aforementioned results showed that CAR crystalline structure was evident. Similar results of obtaining crystalline nanosuspension were observed by Raju et al working on nevirapine..$^{55}$ 


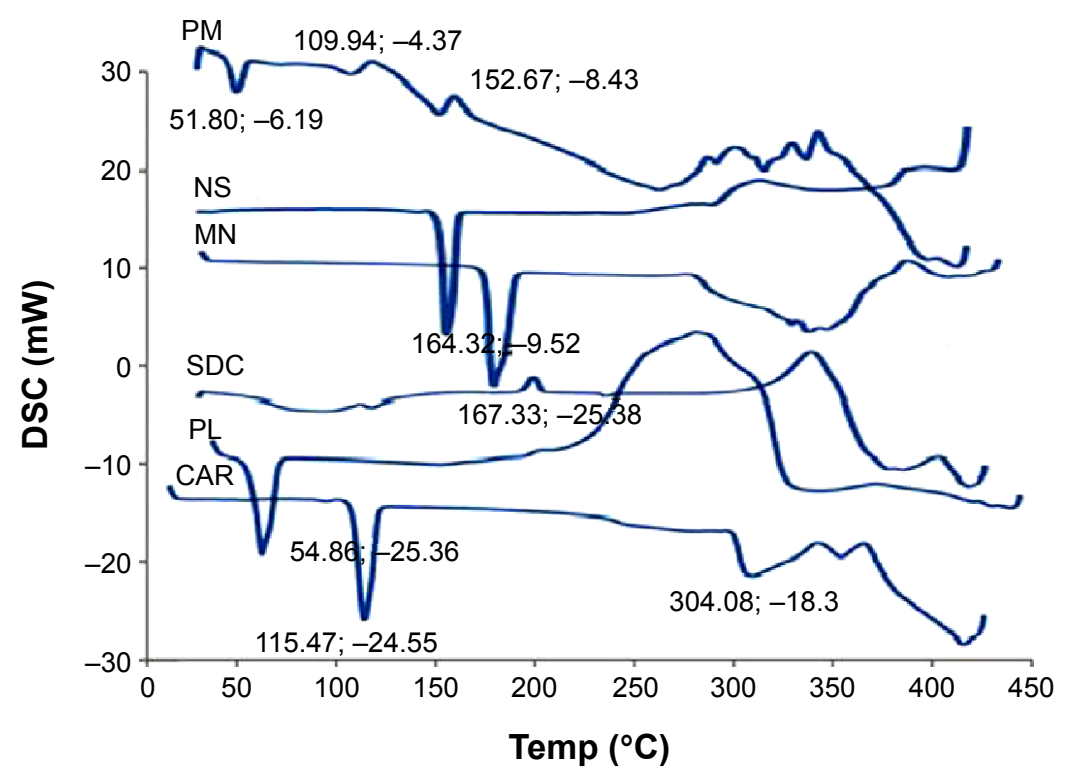

Figure 6 Differential scanning calorimetry (DSC) curves of carvedilol (CAR), Pluronic FI27 (PL), sodium deoxycholate (SDC), mannitol (MN), physical mixture (PM), and lyophilized nanosuspension (NS).

\section{Saturated solubility and in vitro dissolution of optimized nanosuspension formula}

The saturated solubility of the optimized nanosuspension formula showed a significant increase $(P<0.05)$ in solubility (26\%) compared with the coarse CAR powder. The coarse drug demonstrated a saturated solubility of $30.29 \mu \mathrm{g} / \mathrm{mL}$ while the nanosuspension formula showed a saturated solubility of $38.20 \mu \mathrm{g} / \mathrm{mL}$. However, it is worth mentioning that Van Eerdenbrugh et $\mathrm{al}^{56}$ reported that significant increases in solubility due to increased curvature of drug nanoparticles

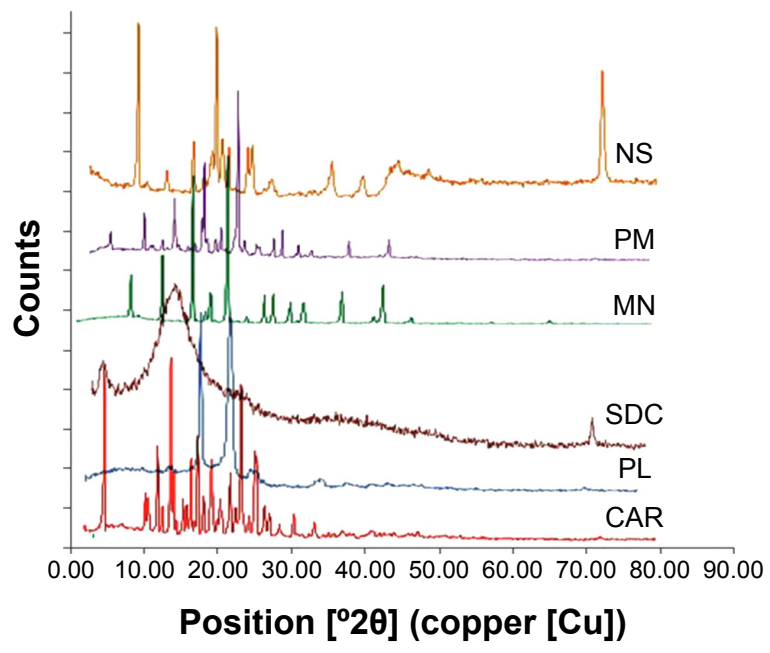

Figure 7 Powder X-ray diffraction patterns of carvedilol (CAR), Pluronic FI27 (PL), sodium deoxycholate (SDC), mannitol (MN), physical mixture (PM), and lyophilized nanosuspension (NS). is not expected with drug nanosizing, rather rapid dissolution kinetics is expected to significantly increase with PS reduction. $^{7}$

The lyophilized nanosuspension formula showed a significant increase in the rate and extent of dissolution and the dissolution rate was maintained at higher level throughout all time intervals compared with the coarse CAR powder (Figure 8). Within 10 minutes, almost $76.63 \%$ of CAR was dissolved from the nanosuspension formula compared with only $24.11 \%$ for the coarse CAR powder, respectively. After 60 minutes, the dissolution was complete $(\approx 100.00 \%)$ for the nanosuspension formula compared with only $44.82 \%$ for the coarse CAR powder, respectively. MDT was equal to 8.94 minutes in the case of coarse drug powder. The optimized nanosuspension formula showed a significant decrease

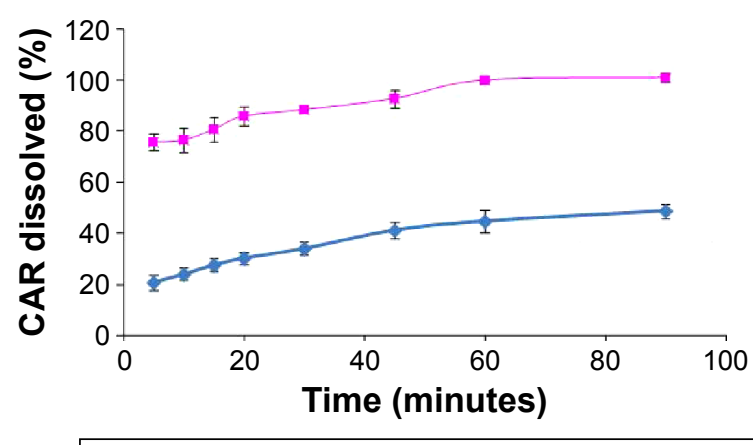

- Lyophilized nanosuspension $\multimap$ Coarse drug

Figure 8 Percentage of CAR dissolved from optimized nanosuspension formula compared to the coarse drug. Abbreviation: CAR, carvedilol. 
in MDT to be 5.26 minutes $(P<0.05)$. The $f 2$ was calculated to compare the dissolution profiles of the coarse drug powder and the optimized nanosuspension formula and was found to be 14.24. Being lower than 50, this indicates the presence of significant difference in the release profiles. ${ }^{38}$

The increased surface area described by the Noyes Whitney equation ${ }^{57}$ and higher surface to volume ratio enabled hydration over larger surface area, and consequently resulted in increased drug dissolution. ${ }^{58}$ Moreover, the increase in dissolution rate caused due to PS reduction can be explained by the decrease in diffusion layer thickness. According to the Prandtl boundary layer equation for flow passing a flat surface, the hydrodynamic boundary layer thickness $\left(\mathrm{h}_{\mathrm{H}}\right)$ can be expressed by Equation 4 as follows:

$$
\mathrm{h}_{\mathrm{H}}=\mathrm{k}\left(\mathrm{L}^{1 / 2} / \mathrm{V}^{1 / 2}\right)
$$

where $\mathrm{L}$ is the length of the surface in the direction of flow, $\mathrm{k}$ denotes a constant, and $\mathrm{V}$ is the relative velocity of the flowing liquid against the flat surface. It is believed that a difference in particle diameter could correspond to a difference in the parameter L. ${ }^{59}$ Bisrat and Nyström have shown that, for solids dispersed in a liquid medium under agitation, a decrease in PS probably leads to a decrease in both $\mathrm{L}$ and $\mathrm{V} .{ }^{60}$ The net effect is reduced $\mathrm{h}_{\mathrm{H}}{ }^{61}$ This decrease in diffusional thickness $\mathrm{h}$ leads to an increase in the concentration gradient $(\mathrm{Cs}-\mathrm{Ct}) / \mathrm{h}$, which consequently increases the dissolution rate. ${ }^{27}$

\section{In vitro simulated delivery and aerodynamic characterization}

The same HPLC method adopted for the determination of total emitted dose (TED) was also used for the evaluation of aerodynamic particle characterization. The method indicated that CAR retention time was 2.1 minutes. The limit of detection was $0.3 \mathrm{ppm}$ and the lower limit of quantification was $0.99 \mathrm{ppm}$. The results of the aerodynamic characterization are shown in Table 5. The lyophilized nanosuspension resulted in significantly smaller MMAD and higher FPD, fine particle fraction, TED, and TED $\%$ than the coarse drug powder $(P<0.05)$.

The smaller MMAD of the lyophilized nanosuspension $(2.80 \mu \mathrm{m})$ compared with coarse drug powder $(4.20 \mu \mathrm{m})$ is expected to result in better lung deposition when used by the patient. ${ }^{62}$ In addition, the TED was higher in the lyophilized nanosuspension suggesting that the nanoparticles flow behavior was better than the coarse drug powder. Hence, the use of CAR in the form of aerosolized nanoparticles using
Table 5 The aerodynamic characteristics of coarse CAR powder and CAR nanoparticles

\begin{tabular}{|c|c|c|}
\hline Parameter & $\begin{array}{l}\text { Coarse drug } \\
(\text { mean } \pm \text { SD) }\end{array}$ & $\begin{array}{l}\text { Nanoparticles } \\
(\text { mean } \pm \text { SD) }\end{array}$ \\
\hline Total emitted dose ( $\mu \mathrm{g} ; \mathrm{TED})$ & $1,472.00 \pm 312.00$ & $\mathrm{I}, 794.00 \pm 178.00$ \\
\hline $\begin{array}{l}\text { Total emitted dose } \% \\
\text { (\% of nominal dose; TED\%) }\end{array}$ & $73.60 \pm 15.60$ & $89.70 \pm 8.90$ \\
\hline Fine particle dose ( $\mu$ g; FPD) & $818.40 \pm 94.20$ & $\mathrm{I}, 469.30 \pm 77.10$ \\
\hline $\begin{array}{l}\text { Fine particle fraction } \\
\text { (\% of emitted dose; FPF) }\end{array}$ & $55.60 \pm 6.40$ & $81.90 \pm 4.30$ \\
\hline $\begin{array}{l}\text { Mass median aerodynamic } \\
\text { diameter }(\mu \mathrm{m} ; \mathrm{MMAD})\end{array}$ & $4.20 \pm 1.50$ & $2.80 \pm 1.20$ \\
\hline $\begin{array}{l}\text { Geometric standard } \\
\text { deviation (GSD) }\end{array}$ & $2.10 \pm 0.40$ & $1.70 \pm 0.50$ \\
\hline
\end{tabular}

Abbreviations: CAR, carvedilol; SD, standard deviation.

mannitol as a coarse carrier is expected to result in better lung deposition and higher bioavailability because of the smaller PS and consequently higher dissolution rate.

The obtained value of MMAD of the inhaled nanoparticles powder $(2.80 \mu \mathrm{m})$ may be attributed to the action of mannitol as a coarse carrier. Mannitol formed a large crystalline matrix outside the nanoparticles during freeze-drying as shown in the scanning electron micrograph (Figure 5), which may have resulted in a bimodal disperse system. Therefore, mannitol has increased the MMAD of the inhaled CAR nanoparticles. ${ }^{63,64}$ This complies with the reported data showing that the use of coarse excipients in dry powder inhalation formulations will increase and inevitably improve particle aerodynamic behavior and flow properties. ${ }^{65}$

\section{Conclusion}

The results of modeling and optimization of CAR nanosuspensions followed by lyophilization in the presence of mannitol (which acted as cryoprotectant and coarse carrier for nanoparticles) showed the possibility of controlling the production of nanoparticles with tailored aerodynamic characteristics suitable for pulmonary delivery. Selection of appropriate surface stabilizers with varying contents of hydrophilic and hydrophobic moieties such as Pluronic F127, F68, or P123 at the optimum ratio of drug to stabilizer enabled the formulation of stable nanosuspensions. Both hydrophobicity and hydrophilicity of Pluronics are essential to obtain stable polymer adsorption onto the nanoparticles as evidenced by the effects of the ratio of hydrophilic to hydrophobic units on the output parameters. Moreover, data mining technologies in the form of ANNS and genetic algorithm proved to be valuable tools for modeling and optimization of CAR nanosuspensions. The aerodynamic properties proved that the optimized formula was a successful 
candidate for pulmonary delivery. However, further in vivo investigations are required to establish the superiority of the developed aerosolized CAR nanoparticles over the existing oral medications of CAR.

\section{Acknowledgment}

Special thanks and gratitude are to be paid to the late Dr Elizabeth Colbourn, the founder and former director of Intelligensys, Co. Ltd, UK, for her unforgettable help and support in using INForm.

\section{Disclosure}

The authors report no conflicts of interest in this work.

\section{References}

1. Bosquillon C, Lombry C, Preat V, Vanbever R. Influence of formulation excipients and physical characteristics of inhalation dry powders on their aerosolization performance. J Control Release. 2001;70(3):329-339.

2. Varshosaz J, Taymouri S, Hamishehkar H. Fabrication of polymeric nanoparticles of poly(ethylene-co-vinyl acetate) coated with chitosan for pulmonary delivery of carvedilol. J Appl Polym Sci. 2014;131(1):39694 (39691-39698).

3. Ali AMA, Abdelrahim MEA. Modeling and optimization of terbutaline emitted from a dry powder inhaler and influence on systemic bioavailability using data mining technology. J Pharm Innov. 2014;9:38-47.

4. Mansour HM, Rhee YS, Wu X. Nanomedicine in pulmonary delivery. Int J Nanomed. 2009;4:299-319.

5. Xi J, Longest PW. Effects of oral airway geometry characteristics on the diffusional deposition of inhaled nanoparticles. J Biomech Eng. 2008;130(1):011008.

6. Salem H, Abdelrahim M, Eid KA, Sharaf M. Nanosized rods agglomerates as a new approach for formulation of a dry powder inhaler. Int J Nanomed. 2011;6:311-320.

7. Abdelbary AA, Li X, El-Nabarawi M, Elassasy A, Jasti B. Comparison of nanomilling and coprecipitation on the enhancement of in vitro dissolution rate of poorly water-soluble model drug aripiprazole. Pharm Dev Technol. 2014;19(4):491-500.

8. Keck CM, Muller RH. Drug nanocrystals of poorly soluble drugs produced by high pressure homogenisation. Eur J Pharm Biopharm. 2006; 62(1):3-16.

9. Van Eerdenbrugh B, Van den Mooter G, Augustijns P. Top-down production of drug nanocrystals: nanosuspension stabilization, miniaturization and transformation into solid products. Int J Pharm. 2008; 364(1):64-75.

10. de Waard H, Hinrichs WL, Frijlink HW. A novel bottom-up process to produce drug nanocrystals: controlled crystallization during freezedrying. J Control Release. 2008;128(2):179-183.

11. Zhang HX, Wang JX, Zhang ZB, Le Y, Shen ZG, Chen JF. Micronization of atorvastatin calcium by antisolvent precipitation process. Int J Pharm. 2009;374(1-2):106-113.

12. Michal EM, Margaret AH, Keith PJ, OWI R. Drug nanoparticles by antisolvent precipitation mixing energy. Langmuir. 2006;22:8951-8959.

13. Abbasi S, Afrasiabi A, Zarchi AAK, Faramarzi MA, Tavoosidana G, Amani A. Preparation and optimization of $\mathrm{N}$-acetylcysteine nanosuspension through nanoprecipitation: an artificial neural networks study. J Pharm Innov. 2014;9:115-120.

14. Rowe RC, Roberts RJ. Intelligent Software for Product Formulation. London: Taylor and Francis; 1998.

15. Sun Y, Peng Y, Chen Y, Shukla AJ. Application of artificial neural networks in the design of controlled release drug delivery systems. $A d v$ Drug Deliv Rev. 2003;55(9):1201-1215.
16. Bas D, Boyaci I. Modeling and optimization II: comparison of estimation capabilities of response surface methodology with artificial neural networks in a biochemical reaction. J Food Eng. 2007;78(3):846-854.

17. Chen Y, Thosar SS, Forbess RA, Kemper MS, Rubinovitz RL, Shukla AJ. Prediction of drug content and hardness of intact tablets using artificial neural network and near-infrared spectroscopy. Drug Dev Ind Pharm. 2001;27(7):623-631.

18. Hussain AS, Yu XQ, Johnson RD. Application of neural computing in pharmaceutical product development. Pharm Res. 1991;8(10): $1248-1252$.

19. Aksu B, Paradkar A, de Matas M, Ozer O, Guneri T, York P. Quality by design approach: application of artificial intelligence techniques of tablets manufactured by direct compression. AAPS Pharm Sci Tech. 2012; 13(4):1138-1146.

20. Liu D, Xu H, Tian B, et al. Fabrication of carvedilol nanosuspensions through the anti-solvent precipitation-ultrasonication method for the improvement of dissolution rate and oral bioavailability. AAPS Pharm Sci Tech. 2012;13(1):295-304.

21. Kramer BK, Ress KM, Erley CM, Risler T. Pharmacokinetic and blood pressure effects of carvedilol in patients with chronic renal failure. Eur J Clin Pharmacol. 1992;43(1):85-88.

22. von Mollendorff E, Reiff K, Neugebauer G. Pharmacokinetics and bioavailability of carvedilol, a vasodilating beta-blocker. Eur J Clin Pharmacol. 1987;33(5):511-513.

23. Decker S, Gabel R-d, Lapotnikoff J, Wirl A, Zimmermann I, Inventors; assignee. Carvedilol-lipophilic solutions. United States patent US 20010036960. 2001.

24. Wen X, Tan F, Jing Z, Liu Z. Preparation and study the 1:2 inclusion complex of carvedilol with beta-cyclodextrin. J Pharm Biomed Anal. 2004;34(3):517-523.

25. Wei L, Li J, Guo L, et al. Investigations of a novel self-emulsifying osmotic pump tablet containing carvedilol. Drug Dev Ind Pharm. 2007; 33(9):990-998.

26. Planinsek O, Kovacic B, Vrecer F. Carvedilol dissolution improvement by preparation of solid dispersions with porous silica. Int J Pharm. 2011; 406(1-2):41-48.

27. Elsayed I, Abdelbary AA, Elshafeey AH. Nanosizing of a poorly soluble drug: technique optimization, factorial analysis, and pharmacokinetic study in healthy human volunteers. Int J Nanomed. 2014;9: 2943-2953.

28. Duret C, Wauthoz N, Sebti T, Vanderbist F, Amighi K. New inhalationoptimized itraconazole nanoparticle-based dry powders for the treatment of invasive pulmonary aspergillosis. Int J Nanomed. 2012;7: 5475-5489.

29. Ali AA, Ali AM. Optimization of propranolol $\mathrm{HCl}$ release kinetics from press coated sustained release tablets. Pharm Dev Technol. 2013;18(5): $1238-1246$

30. Rowe RC, Colbourn EA. Data mining methods in pharmaceutical formulation. In: Balakin KV, editor, Pharmaceutical Data Mining: Approaches and Applications for Drug Discovery. Hoboken, NJ: John Wiley \& Sons, Inc.; 2009:401-421.

31. Plumb AP, Rowe RC, York P, Brown M. Optimisation of the predictive ability of artificial neural network (ANN) models: a comparison of three ANN programs and four classes of training algorithm. Eur J Pharm Sci. 2005;25(4):395-405.

32. Thakkar HP, Patel BV, Thakkar SP. Development and characterization of nanosuspensions of olmesartan medoxomil for bioavailability enhancement. J Pharm Bioallied Sci. 2011;3(3):426-434.

33. Gao B, Wang J, Wang D, et al. A novel preparation method for drug nanocrystals and characterization by ultrasonic spray-assisted electrostatic adsorption. Int J Nanomed. 2013;8:3927-3935.

34. Sun J, Wang F, Sui Y, et al. Effect of particle size on solubility, dissolution rate, and oral bioavailability: evaluation using coenzyme Q10 as naked nanocrystals. Int J Nanomed. 2012;7:5733.

35. Rahman Z, Zidan AS, Khan MA. Risperidone solid dispersion for orally disintegrating tablet: its formulation design and non-destructive methods of evaluation. Int J Pharm. 2010;400(1):49-58. 
36. Hecq Jrm, Deleers M, Fanara D, Vranckx H, Amighi K. Preparation and characterization of nanocrystals for solubility and dissolution rate enhancement of nifedipine. Int J Pharm. 2005;299(1):167-177.

37. Raju V, Murthy KV. Development and validation of new discriminative dissolution method for carvedilol tablets. Indian J Pharm Sci. 2011; 73(5):527-536.

38. Costa P, Sousa Lobo JM. Modeling and comparison of dissolution profiles. Eur J Pharm Sci. 2001;13(2):123-133.

39. British Pharmacopoeia. Preparations for inhalation. Aerodynamic assessment of fine particles-fine particle dose and particle size distribution (Ph. Eur. method 2.9.18). In: British Pharmacopoeia, ed. Vol 4. London: British Pharmacopoeia; 2005:A277-A290.

40. European Pharmacopeia. 2.9.18: Preparations for inhalation: aerodynamic assessment of fine particles. In: European Pharmacopeia. Strasbourg, France: Council of Europe; 2002:209-217.

41. United States Pharmacopeia. Aerosols, nasal sprays, metered dose inhalers and dry powder inhalers. The United States pharmacopeia 28 [and] The national formulary 23. Rockville, MD: The Board of Trustees; 2005:2359-2377.

42. Basaveswara Rao MV, Nagendrakumar AVD, Yedukondalu M, Raman BV. New validated rp-hplc method for the estimation of carvedilol in pharmaceutical formulation. Int J Pharm Sci. 2012;4(2): 253-358.

43. Copley Scientific Limited. Copley Scientific Catalogue. Nottingham: Copley Scientific Limited; 2015. Available from: http://www. copleyscientific.com/files/ww/brochures/Inhaler\%20Brochure $\% 20$ 2015_Rev4\%20_Low\%20Res_.pdf. Accessed September 17, 2015.

44. Van Oort M. In vitro testing of dry powder inhalers. Aerosol Sci Tech. 1995;22(4):364-373.

45. Ghaffari A, Abdollahi H, Khoshayand M, Bozchalooi IS, Dadgar A, Rafiee-Tehrani M. Performance comparison of neural network training algorithms in modeling of bimodal drug delivery. Int J Pharm. 2006; 327(1):126-138

46. Aghajani M, Shahverdi AR, Amani A. The use of artificial neural networks for optimizing polydispersity index (PDI) in nanoprecipitation process of acetaminophen in microfluidic devices. AAPS Pharm Sci Tech. 2012;13(4):1293-1301.

47. Schroen CGPH, Cohen Stuart MA, Maarschalk KV, van der Padt A, van't Riet K. Influence of preadsorbed block copolymers on protein adsorption: Surface properties, layer thickness, and surface coverage. Langmuir. 1995;11:3068-3074.

48. Nejadnik MR, Olsson AL, Sharma PK, van der Mei HC, Norde W, Busscher HJ. Adsorption of pluronic F-127 on surfaces with different hydrophobicities probed by quartz crystal microbalance with dissipation. Langmuir. 2009;25(11):6245-6249.

49. Li J-T, Caldwell KD, Rapoport N. Surface properties of pluronic-coated polymeric colloids. Langmuir. 1994;10:4475-4482.

50. Besheer A, Vogel J, Glanz D, Kressler J, Groth T, Mader K. Characterization of PLGA nanospheres stabilized with amphiphilic polymers: hydrophobically modified hydroxyethyl starch vs pluronics. Mol Pharm. 2009;6(2):407-415.
51. Abdelbary AA, Li X, El-Nabarawi M, Elassasy A, Jasti B. Effect of fixed aqueous layer thickness of polymeric stabilizers on zeta potential and stability of aripiprazole nanosuspensions. Pharm Dev Technol. 2013; 18(3):730-735.

52. Bendas ER, Abdelbary AA. Instantaneous enteric nano-encapsulation of omeprazole: pharmaceutical and pharmacological evaluation. Int J Pharm. 2014;468(1-2):97-104.

53. Al-Mahallawi AM, Abdelbary AA, Aburahma MH. Investigating the potential of employing bilosomes as a novel vesicular carrier for transdermal delivery of tenoxicam. Int J Pharm. 2015;485(1-2):329-340.

54. Hildesheim J, Finogueev S, Aronhime J, Dolitzky B-Z, Ben-Valid S, Kor I, Inventors; assignee. Carvedilol United States patent US 7056942 B2. 2006.

55. Raju A, Reddy AJ, Satheesh J, Jithan AV. Preparation and Characterisation of nevirapine oral nanosuspensions. Ind J Pharm Sci. 2014;76(1): $62-71$

56. Van Eerdenbrugh B, Vermant J, Martens JA, et al. Solubility increases associated with crystalline drug nanoparticles: methodologies and significance. Mol Pharm. 2010;7(5):1858-1870.

57. Kesisoglou F, Panmai S, Wu Y. Nanosizing-oral formulation development and biopharmaceutical evaluation. Adv Drug Deliv Rev. 2007; 59(7):631-644.

58. Dolenc A, Kristl J, Baumgartner S, Planinsek O. Advantages of celecoxib nanosuspension formulation and transformation into tablets. Int J Pharm. 2009;376(1-2):204-212.

59. Mosharraf M, C. N. The effect of particle size and shape on the surface specific dissolution rate of microsized practically insoluble drugs. Int J Pharm. 1995;122(1-2):35-47.

60. Bisrat M, C. N. Physicochemical aspects of drug release. VIII. The relation between particle size and surface specific dissolution rate in agitated suspensions. Int J Pharm. 1988;47(1-3):223-231.

61. Niebergall PJ, Milosovich G, Goyan JE. Dissolution rate studies. II. Dissolution of particles under conditions of rapid agitation. J Pharm Sci. 1963;52:236-241.

62. Yang W, Johnston KP, Williams III RO. Comparison of bioavailability of amorphous versus crystalline itraconazole nanoparticles via pulmonary administration in rats. Eur J Pharm Biopharm. 2010;75(1):33-41.

63. Kaialy W, Alhalaweh A, Velaga SP, Nokhodchi A. Effect of carrier particle shape on dry powder inhaler performance. Int J Pharm. 2011; 421(1):12-23.

64. Rattanupatam T, Srichana T. Budesonide dry powder for inhalation: effects of leucine and mannitol on the efficiency of delivery. Drug Deliv. 2014;21(6):397-405.

65. Pilcer G, Amighi K. Formulation strategy and use of excipients in pulmonary drug delivery. Int J Pharm. 2010;392(1):1-19.
International Journal of Nanomedicine

\section{Publish your work in this journal}

The International Journal of Nanomedicine is an international, peerreviewed journal focusing on the application of nanotechnology in diagnostics, therapeutics, and drug delivery systems throughout the biomedical field. This journal is indexed on PubMed Central, MedLine, CAS, SciSearch $®$, Current Contents $\AA /$ Clinical Medicine,

\section{Dovepress}

Journal Citation Reports/Science Edition, EMBase, Scopus and the Elsevier Bibliographic databases. The manuscript management system is completely online and includes a very quick and fair peer-review system, which is all easy to use. Visit http://www.dovepress.com/ testimonials.php to read real quotes from published authors. 